Jacques Freyssinet, Négocier l'emploi. 50 ans de négociations interprofessionnelles sur l'emploi et la formation

Paris, Éditions Liaisons, coll. « Liaisons sociales », 2010

\title{
Vincent Gayon
}

\section{OpenEdition}

Journals

Édition électronique

URL : http://journals.openedition.org/travailemploi/5388

DOI : 10.4000/travailemploi.5388

ISSN : 1775-416X

Éditeur

DARES - Ministère du Travail

Édition imprimée

Date de publication : 15 septembre 2011

Pagination : 86-88

ISSN : 0224-4365

\section{Référence électronique}

Vincent Gayon, « Jacques Freyssinet, Négocier l'emploi. 50 ans de négociations interprofessionnelles sur l'emploi et la formation », Travail et Emploi [En ligne], 127 | juillet-septembre 2011, mis en ligne le 18 mai 2012, consulté le 22 septembre 2020. URL : http://journals.openedition.org/travailemploi/5388 ; DOI https://doi.org/10.4000/travailemploi.5388

Ce document a été généré automatiquement le 22 septembre 2020.

(c) Direction de l'animation de la recherche, des études et des statistiques (Dares) 


\section{Jacques Freyssinet, Négocier l'emploi. 50 ans de négociations interprofessionnelles sur l'emploi et la formation}

Paris, Éditions Liaisons, coll. « Liaisons sociales », 2010

Vincent Gayon

\section{RÉFÉRENCE}

Jacques Freyssinet, Négocier l'emploi. 50 ans de négociations interprofessionnelles sur l'emploi et la formation, Paris, Éditions Liaisons, coll. «Liaisons sociales », 2010

1 Négocier l'emploi offre une analyse d'un demi-siècle de négociations interprofessionnelles sur l'emploi en France. Jacques Freyssinet ne se livre pas à une genèse des " politiques de l'emploi », catégorie administrative d'apparition récente qui supplante dans les années 1960 la "politique de la main d'œuvre »1. Son objet est bien la négociation interprofessionnelle sur l'emploi qui se développe au départ pour gérer les restructurations industrielles et met aux prises trois types d'acteurs : l'État, les patronats, les syndicats. Plus généralement, son objectif est de rendre compte de la dynamique des accords signés entre 1958 et 2008 « qui font référence à l'emploi comme objectif de la négociation et source de légitimation de leur contenu» (p. 11). L'emploi est défini d'une triple manière par l'auteur: l'état du salarié, l'objet du pouvoir de direction de l'employeur, l'objectif des négociations (préservation ou création d'emplois). Cette définition composite, issue de travaux en droit social ${ }^{2}$, est opératoire pour cerner «la rupture historique intervenue dans la nature des négociations collectives. Aussi longtemps que durent la croissance forte et le plein emploi, la négociation peut mener de pair la construction du statut de salarié et la poursuite d'objectifs relatifs à l'emploi»(p. 16). A l'opposé, avec un chômage massif, la 
négociation collective sur l'emploi se disjoint: le statut protecteur associé au travail apparaît pour le patronat et pour l'État, voire pour certains syndicats, comme le frein à la création d'emplois et à la compétitivité.

2 L'analyse s'organise selon deux axes. D'abord, une entrée synthétique autour de questions transversales : quels ont été les facteurs d'émergence des négociations sur l'emploi, quelle est la place de la négociation interprofessionnelle dans le système global de régulation de l'emploi, quel est l'impact exercé par l'environnement international. Ensuite, une entrée plus historique dégageant les trois ou quatre configurations successives (et en partie enchâssées) des négociations sur l'emploi. La première phase débute par l'accord national interprofessionnel du 31 décembre 1958 et s'achève dans le milieu des années 1970. Elle est marquée par la conquête de nouveaux droits sociaux et par l'invention ou la consolidation d'instruments de gestion des conséquences des restructurations industrielles: indemnisation du chômage, mais aussi reclassements, formations professionnelles, préretraite. Dans la deuxième séquence - entre le milieu de la décennie 1970 et le début des années 1980 -, le chômage massif s'il est encore perçu comme transitoire et cyclique dans une perspective keynésienne, pousse cette fois à la mise en place de moyens financiers exceptionnels pour en atténuer les coûts sociaux (revenus de substitution). Ce qui a pour effet de gonfler les budgets sociaux. Le taux de chômage élevé, devenu chronique, et son interprétation économique désormais "structurelle ", la troisième phase de négociations collectives - depuis les années 1980 jusqu'aux années 2000 - se caractérise par des discussions plus fragmentées portant sur tels «freins » ou « obstacles » pesant sur le seul marché du travail : formation continue, durée du travail, assouplissement des contrats de travail, etc. Les conquêtes sociales ne sont plus considérées comme une solution ou un palliatif mais bien comme la base du problème lui-même. Enfin, l'accord du 11 janvier 2008 sur la «modernisation du marché du travail » marque pour Jacques Freyssinet une forme de retour à une approche multidimensionnelle de l'emploi alliant l'analyse de ses déterminants, des statuts et des modes de gestion, ainsi que la reconnaissance au moins formelle par l'État d'une « sphère de responsabilité autonome dans la régulation de l'emploi » (p. 10); sphère de responsabilité qui a très tôt exclu de son domaine de compétences, dans la période « planiste-keynésienne » comme dans la période "désinflationniste-néolibérale ", toute négociation sur les salaires, sur la politique (macro)économique et la politique industrielle.

3 On le voit, le champ couvert par l'ouvrage est vaste et son propos ambitieux. Il embrasse un objet d'études aride sur une assez longue période et se situe à la croisée de différents domaines de connaissance : histoire de l'État social et du droit social, histoire de la représentation syndicale et patronale, mais aussi, en toile de fond, histoire économique et, à un moindre degré, histoire des savoirs économiques sur le chômage et l'emploi. L'auteur ne travaille pas sur les archives des confédérations ou par entretiens. Son analyse repose sur une connaissance pratique des acteurs de ces négociations, sur une lecture aiguisée des bulletins des centrales syndicales et patronales et sur la littérature spécialisée. S'il ne se livre pas à une sociologie des négociateurs parties prenantes (logiques de recrutement, trajectoires sociales, élévation du niveau de qualification, multipositionnalité, etc.), il se garde bien de les homogénéiser. "L'État ", « le patronat » et "les syndicats » sont en bien des cas conçus de manière désagrégée, permettant de voir les conflits qui les traversent et les logiques d'action spécifiques et évolutives que leurs membres suivent, ainsi que les propositions qu'ils entendent 
défendre en amont, au cours ou après les négociations. Se dessine d'ailleurs un enjeu tacite de ces négociations interprofessionnelles que l'auteur évoque finalement peu. Elles sont un des lieux propices à la représentation ou à la mise en forme sociale du " salariat ", du «patronat » ou de "l'État ", où ces associés-rivaux s'entendent déjà $a$ minima sur la capacité des autres à faire exister - et à agir au nom de - ces acteurs collectifs réifiés. Les protagonistes (État y compris) sont donc à ce titre des «partenaires sociaux » mais dans un sens sociologique, éloigné de l'usage que font les acteurs de cette expression.

4 C'est la dynamique des négociations qui intéresse l'auteur, parfois sur des périodes très resserrées. Plutôt qu'une analyse strictement économique cherchant à isoler l'hypothétique variable « macro » agissante, la démarche suivie privilégie l'hybridation d'approches juridiques, sociologiques et économiques pour expliquer comment les enjeux des négociations, les priorités défendues, les intérêts respectifs et la conjoncture économique elle-même se construisent et se redéfinissent en situation dans des jeux contraignants. Plutôt qu'une théorie de la négociation collective, l'auteur défend plus modestement une « méthode » d'analyse (p. 18). Néanmoins cette méthode engage bien une théorie qui rompt de facto avec tout mécanicisme ou déterminisme économique et se rattache à une forme de socioéconomie intéressée par la «complexité des interactions entre action publique et négociations collectives » (p. 234).

5 L'analyse de l'accord du 11 janvier 2008 est très éclairante à cet égard. L'auteur met d'abord l'accent sur la structuration en groupes de travail mêlant syndicats et patronats, puis intègre les temporalités présidentielle et gouvernementale voire aussi européenne, et celle liée à une série de rapports d'expertise publiés dans la période. Ensuite, la dynamique de négociation proprement dite montre combien les positions évoluent entre un patronat unifié et maximaliste autour de ses « quarante propositions pour moderniser le Code du travail » et des syndicats au départ éclatés (la CGT ne signera pas l'accord final). Négocié au pas de charge en quatre mois sur des domaines étendus (entrée et évolution dans l'emploi, sortie et retour à l'emploi), marqué par des moments de tension sur des "points durs" (période d'essai, rupture à l'amiable, contrat de projet, portabilité/transférabilité), cet accord est le produit de conflits et de compromis dont chaque protagoniste retiendra ce qu'il y est parvenu à inscrire, à sauver, à gagner, etc. Le détour par la production de cet accord enrichit donc considérablement l'analyse de son contenu à laquelle se livre dans un second temps $\mathrm{J}$. Freyssinet, en suivant les lignes de force précédemment dégagées. Ce dernier peut ainsi conclure que : «L'harmonie formelle des textes législatifs qui règlent [les] rapports [entre l'État, les syndicats et le patronat] ne saurait masquer l'étroit et opaque entremêlement de leurs stratégies. » (p. 232)

6 En dressant l'évolution d'ensemble de la négociation interprofessionnelle, l'auteur montre bien que rien n'est écrit à l'avance, que des ajustements se réalisent de manière incrémentale et incertaine au cours même des négociations, que des zones de tractations perçues comme restreintes ou mineures sur l'instant en sont venues à compter et à imprimer une tendance de fond à l'ensemble du mode de régulation de l'emploi. Sont ainsi restitués souvent dans le détail le contenu des propositions et des résultats des négociations, ainsi que les spécificités des conjonctures de négociation : en particulier, les enjeux tactiques pour l'État à les ouvrir, les accompagner ou les valider, puis pour les patronats ou les syndicats à se présenter comme interlocuteur nécessaire, responsable, opposant, ou à jouer l'offensive, la défensive ou l'ouverture, etc. Le lecteur 
exigeant y fera son miel, le lecteur pressé pourra compter sur une argumentation structurée comprenant de toniques conclusions.

7 Même si on aurait pu souhaiter une plus forte systématisation sociologique, sont mis à jour les enjeux de constitution de cet espace labile de confrontations, soumis aux diverses stratégies, composé en de multiples « niveaux » de négociation (national, de branche, dans l'entreprise) et fragmenté en divers dossiers toujours plus techniques. Alors même que le caractère multidimensionnel du problème de l'emploi est reconnu par l'ensemble des protagonistes, leurs logiques d'action respectives les poussent au contraire - dès qu'il est question d' « accords de contenu » - à le confiner, le découper, dans une logique des petits pas, par crainte de trop concéder, par souci d'entériner certains acquis ou par gestion pragmatique de dispositifs inventés par d'autres. Ce qui explique sans doute comment la doctrine du patron «seul juge » de la bonne gestion de son entreprise s'impose (avec l'aide de la loi et de la jurisprudence), et comment les grands partages entre "politiques économiques" et "politiques sociales", entre " économique » et "politique », entre "syndicats » et "partis", se stabilisent et se soustraient à toute forme de critique indigène. Mais dans cette sectorisation et cette sophistication du problème de l'emploi, quelle responsabilité imputer aussi à la "professionnalisation" des permanents syndicaux et patronaux dans les instances paritaires de collecte, de redistribution et de négociation? Derrière l'immobilité des sigles et des prises de position publiques, n'aurait-il pas fallu insister davantage sur l'évolution sensible des réalités syndicales et patronales ${ }^{3}$ ? A cet égard, un autre élément peu questionné: la restructuration des grands groupes industriels, le développement du commerce intrafirme et de la sous-traitance. Dans quelle mesure la négociation interprofessionnelle a achoppé en ces domaines? Les modalités d'action de ces groupes qui se passent volontiers de la représentation du CNPF, de l'UIMM ou du MEDEF auraient-elles pu fournir une utile mise en contraste ? On s'étonne aussi de ne pas avoir plus d'éléments sur les transformations des élites de l'État qui ont pu gérer ce dossier dans le champ bureaucratique national (par exemple, les enjeux du rattachement des questions d'emploi au ministère de l'Économie en 2007) ou bien, en lien ou au sein d'organisations comme l'UE ou l'OCDE.

8 A l'image du parcours de son auteur, qui a pu être formateur syndical, professeur émérite d'économie à la Sorbonne, président du Conseil administration de l'ANPE, directeur de l'IRES, puis président du conseil scientifique du Centre d'études de l'emploi, l'ouvrage constitue une véritable synthèse des recherches sur cette « zone de contacts » dans la régulation de l'économie française, sans jamais rogner les matières faisant l'objet des négociations, ni la diversité et l'évolution des positions et prises de position des acteurs. L'exercice était ardu, le pari d'ensemble remporté. Dominant la technicité du sujet, l'argumentation se révèle prudente sans néanmoins verser dans les conclusions pusillanimes de "l'expert». Doté d'une solide bibliographie et d'une table chronologique bien utile, l'ouvrage fait déjà office de référence en ce domaine. 


\section{NOTES}

1. D'une gestion de la pénurie qualitative et quantitative de main d'œuvre, on passe avec la politique de l'emploi à «l'ensemble des dispositifs mis en œuvre pour anticiper ou corriger les déséquilibres des marchés du travail ou pour en réduire le coût social. Attribut du ministère du Travail, elle se distingue de ce que l'on peut appeler, au sens large, la " politique pour l'emploi " et qui désigne l'ensemble des interventions publiques visant, à titre principal ou secondaire, à agir sur le niveau et la qualité de l'emploi (p. 25, soulignés par l'auteur), telles que la politique économique, la législation du travail, la protection sociale, l'éducation ou la politique familiale, etc. En la matière, l'auteur avait déjà pu fournir des synthèses : «La diversification des acteurs de la politique de l'emploi (1945-1973)», Travail et emploi, n¹10, avril-mai 2007 ; «L'émergence des politique de l'emploi (1945-1973)» in Alain Chatriot, Odile Join-Lambert, Vincent Viet (dir.), Les politiques du Travail. Acteurs, institutions, réseaux (1906-2006), Rennes, Presses Universitaires de Rennes, 2006. Voir plus récemment, dans une perspective sociologique: Fabrice Colomb, Genèse et transformations des politiques de l'emploi en France. Une histoire des représentations chez l'élite décisionnaire de l'emploi, Université Paris I, thèse de doctorat, 2010 ; ou plus économique : Philippe Askenazy, Les décennies aveugles. Emploi et croissance. 1970-2010, Paris, Seuil, 2011.

2. Ceux en particulier d'Antoine Lyon-Caen et Tamar Katz.

3. On pense aux travaux de sociologie politique du syndicalisme et du patronat curieusement absents : comme ceux de Nicolas Defaud sur la CFDT, de Karel Yon sur la CGT-FO, de Sophie Béroud sur la CGT, d'Anne-Catherine Wagner sur la Confédération européenne des syndicats, de Michel Offerlé sur les organisations patronales, de Sabine Rozier sur le mécénat de "solidarité " des entreprises ou de Hélène Michel sur les lobbyistes européens.

\section{AUTEURS \\ VINCENT GAYON}

Politiste - Maître de conférences à l'Université Paris Dauphine, IRISSO - UMR CNRS 